\title{
Heat transfer analysis in the human abdomen with a focus upon correlation between the amount of visceral fat and skin temperature
}

\author{
K. Shimano ${ }^{1,2}$ \& Y. Shimano ${ }^{1,3}$ \\ ${ }^{1}$ Hutech-Laboratory Co., Japan \\ ${ }^{2}$ Tokyo City University, Japan \\ ${ }^{3}$ Hokusei-kai Medical Co., Japan
}

\begin{abstract}
Obesity can cause diabetes, ischaemic heart disease and cerebrovascular disease. It is important for obese patients and their doctors to keep recognising changes in the amount of visceral fat on a regular basis. However, at the moment, costly CT scanning with radiation exposure is the only option for detailed information about the amount of fat in the abdomen. In this study, feasibility of a noninvasive fat measurement method with a focus upon low thermal conductivity of fat was examined. Using the boundary element method, the authors conducted 2dimensional heat transfer analysis in the abdomens of two volunteers. Abdominal cross section models were developed from CT scanning data, and the skin surface temperature was imposed as boundary conditions. The present computational results strongly suggested a close correlation of the amount of visceral fat with the state of heat transfer and the resultant distribution of skin temperature. Although more investigation is required, the present theory proved promising for development of a new system of fat measurement.
\end{abstract}

Keywords: obesity, visceral fat, thermal conductivity, heat transfer analysis.

\section{Introduction}

Obesity is one of the most serious health problems in modern society as it can result in diabetes, ischaemic heart disease and cerebrovascular disease, which account for more than a quarter of deaths in Japan. Reduction in visceral fat under supervision of a medical doctor is an effective measure for prevention of such disease. However, obese patients are likely to stop making an effort to lose 
weight. It is, therefore, crucial to keep obese patients regularly informed of change in the amount of visceral fat because such information will be a great help in maintaining their motive for weight loss. Measurement of visceral fat is also recognised as an important matter for proper diagnosis [1].

CT scanning is the best option for measurement of the visceral fat as far as accuracy is concerned. Having CT scans on a daily basis is, however, unacceptable due to high cost and radiation exposure. Although a non-invasive technique of fat measurement by bioelectrical impedance has been proposed [2], it is not comparable to CT scanning in terms of accuracy.

Against the background mentioned above, the authors are trying to develop a new technique of visceral fat measurement with a special focus upon heat transfer. The idea is based on thermoregulation and thermal conductivity of fat. Thermoregulation is an ability of the body to keep the internal temperature constant. On the other hand, thermal conductivity represents the property of a material to transmit heat, and fat has a thermal conductivity lower than other bodily parts. It is expected from these two facts that temperature measured on the skin surface reflects the internal distribution of fat.

In this study, heat transfer analysis in abdomens was conducted to examine feasibility of the fat measurement from skin temperature. Computational models of abdominal cross sections were developed from CT images of two volunteers. Distributions of the skin temperature were obtained by infrared thermography and imposed as boundary conditions. The degree of correlation between the internal distribution of fat and the state of heat transfer is discussed.

\section{Heat conduction}

\subsection{Heat conduction equation}

Heat is constantly being generated in the human body by metabolism and released into the surrounding environment through exhalation, evaporation of sweat and heat transfer from the skin surface. Here, the effects of exhalation and evaporation are excluded from the discussion because the human abdomen is considered under the conditions of no perspiration.

Heat generated inside the body is transferred to the skin surface by conduction. Heat flux in the $x$-direction by conduction is proportional to thermal conductivity and temperature gradient in this direction. Fourier's law describes this relationship as follows:

$$
q_{x}=-\lambda \frac{\partial T}{\partial x}
$$

where $q_{x}, \lambda$ and $T$ represent heat flux in the $x$-direction, thermal conductivity and temperature, respectively. Heat flux in another direction is expressed in the same manner as eqn (1). Temperature distribution is governed by the heat conduction equation, which is written in the following form for steady states:

$$
\lambda\left(\frac{\partial^{2} T}{\partial x^{2}}+\frac{\partial^{2} T}{\partial y^{2}}+\frac{\partial^{2} T}{\partial z^{2}}\right)+Q=0
$$


where $Q$ represents heat generation per unit time per unit volume. Note that the first three terms on the lefthand side of eqn (2) are identical to divergence of the heat flux vector $\left(q_{x}, q_{y}, q_{z}\right)$. This means that a balance between incoming heat and outgoing heat is expressed by eqn (2).

\subsection{Effects of fat on heat conduction in abdomen}

Two temperature fields determined by simple 1-dimensional heat conduction are compared in Fig.1. Length $L$, heat flux $q_{x}$, temperature at the left end $T_{0}$ are common to both fields but field (a) has a higher thermal conductivity, i.e. $\lambda_{a}>\lambda_{b}$. It is obvious from eqn (1) that the temperature gradient in field (b) should be steeper than in (a). As a natural consequence of this, temperature at the right end of (b) $T_{b}$ should be lower than $T_{a}$. Application of this 1-dimensional theory to the human abdomen is straightforward. As the internal body temperature is kept constant like $T_{0}$ and fat has a lower thermal conductivity than other portions, an abdomen containing more fat tends to have lower skin temperature. Furthermore, a person having more fat in the abdomen tends to be larger in size. This contributes to further decrease in skin temperature because it is manifest from Fig.1 that a longer $L$ would result in a far lower temperature at the right end.

In more realistic multi-dimensional heat conduction, there is also another tendency that less heat is transferred through a material with a lower thermal conductivity. Simply put, heat "prefers" to go through regions with lower heat resistance. This could be easily comprehended if an analogy between heat flux and electric current were considered.

As explained above, the amount of fat inside the abdomen is expected to have a close correlation with the state of heat transfer and the resultant temperature distribution on the skin surface. More details of heat transfer in the abdomen are investigated in the following chapters with 2-dimensional computational models.

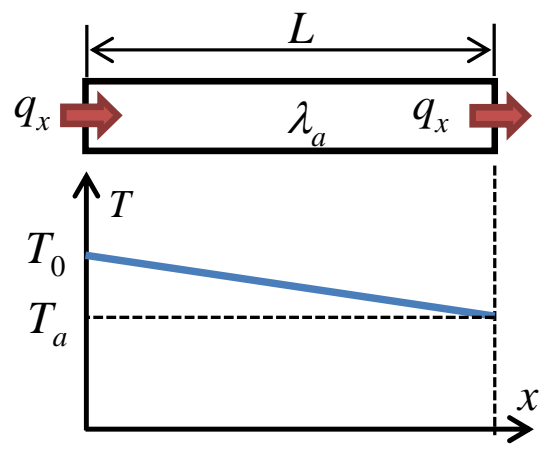

(a) higher conductivity

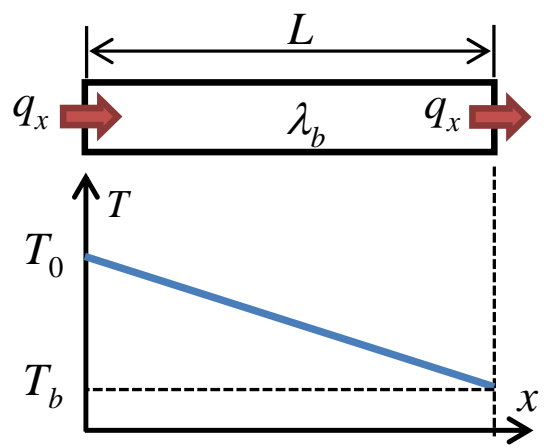

(b) lower conductivity

Figure 1: Comparison of two 1-d temperature fields with different thermal conductivities $\left(\lambda_{a}>\lambda_{b}\right)$. 


\section{Modelling of heat transfer in abdomen}

\subsection{Approach}

2-dimensional heat transfer analysis was carried out on abdominal cross sections including umbilici. Geometric models of abdominal cross sections were made from CT scans of two male volunteers. Temperature distributions imposed as boundary conditions were also obtained from the volunteers by infrared thermography.

The governing equation solved was a 2-dimensional version of the steady heat conduction equation:

$$
\lambda\left(\frac{\partial^{2} T}{\partial x^{2}}+\frac{\partial^{2} T}{\partial y^{2}}\right)+Q_{i n}=0
$$

Equation (3) is consistent with the 3-dimensional heat conduction equation (2) because incoming heat $Q_{\text {in }}$ consists of not only heat generation $Q$ but also heat moving in from other planes, i.e.

$$
Q_{i n}=Q+\lambda \frac{\partial^{2} T}{\partial z^{2}}=Q-\frac{\partial q_{z}}{\partial z}
$$

\subsection{Geometric models}

Figure 2 shows two geometric models made from CT scans. Each model consists of 13 subdomains. Relevant data of the two volunteers is given in Table 1.

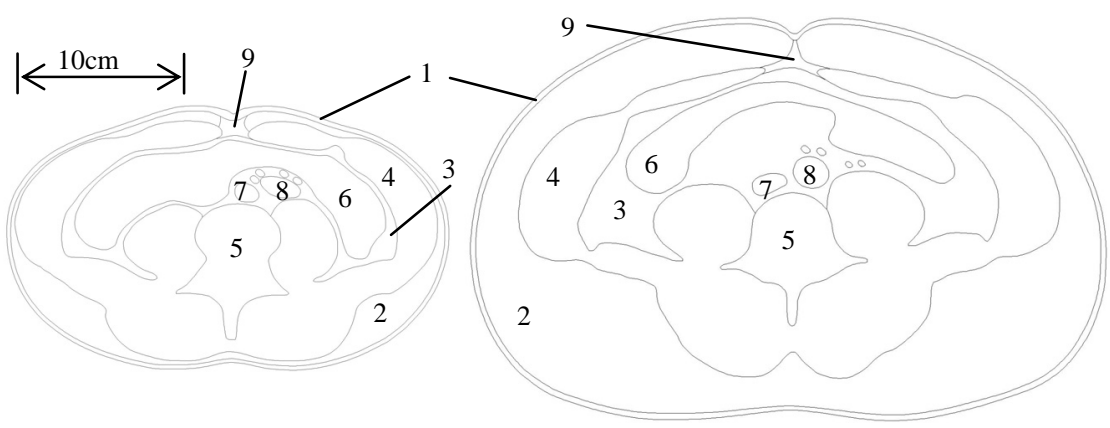

Model A

Model B

Figure 2: $\quad$ Two geometric models made from CT scans. Each subdomain is given a unique ID number as follows: 1 . skin, 2. subcutaneous fat, 3. visceral fat, 4. muscle, 5. vertebra, 6. intestines, 7. aorta, 8. inferior vena cava, 9. fascia, 10. superior mesenteric artery (SMA), 11. superior mesenteric vein (SMV), 12. inferior mesenteric artery (IMA), 13. inferior mesenteric vein (IMV). Small circular regions near subdomain 8 are subdomains 10-13, of which IDs are not written in the figure to avoid overlaying too great a number of IDs. 
Table 1: $\quad$ Relevant data of two male volunteers.

\begin{tabular}{|c|c|c|}
\hline Volunteer & A & B \\
\hline \hline Ethnicity & Japanese & Japanese \\
\hline Age & 21 & 23 \\
\hline Height & $167 \mathrm{~cm}$ & $170 \mathrm{~cm}$ \\
\hline Weight & $52 \mathrm{~kg}$ & $93 \mathrm{~kg}$ \\
\hline $\begin{array}{c}\text { Abdominal } \\
\text { circumference }\end{array}$ & $68 \mathrm{~cm}$ & $102 \mathrm{~cm}$ \\
\hline Subcutaneous fat & $43 \mathrm{~cm}^{2}$ & $305 \mathrm{~cm}^{2}$ \\
\hline Visceral fat & $26 \mathrm{~cm}^{2}$ & $95 \mathrm{~cm}^{2}$ \\
\hline
\end{tabular}

A set of CT scanning data is given in the form of DICOM (digital imaging and communications in medicine). Internal boundaries dividing the whole region into the subdomains were extracted by detecting spots with great spatial change in CT value.

\subsection{Acquisition of temperature distribution on skin surface}

Temperature distributions on the skin surfaces of the two volunteers were measured by thermography.

Measurement was carried out in the morning after 10-hour fasting so that activity of the digestive system could be minimised. The air temperature was kept within a range between 29 and 30 degrees Celsius. The examinees had been in rest with their abdomens exposed to the air for 20 minutes before thermographic images were taken. Thick black curtains surrounded the examinees to avoid complicated effects of radiation from objects in the room. No perspiration was observed throughout the measurement session. Thermographic images were taken from four directions with an interval angle of 90 degrees, i.e. anterior, posterior and two lateral directions.

Figure 3 explains how to obtain the temperature distribution along the circumference of the abdominal cross section including the umbilicus. Temperature at the height of the umbilicus in the thermographic image was projected onto the corresponding position on the circumference as shown in Fig.3. This process was repeated for all the images taken from the four directions. In some positions, two temperature values could be picked up from two thermographic images because the regions covered by the two neighbouring images were partly overlapped. For example, the front right part of the abdominal skin was included in both anterior and right images. Uniqueness of the temperature was assured by connecting temperature curves from different thermographic images without any unnatural gaps.

The circumferential temperature distributions of models A and B are plotted in Figure 4 against $s$, which is a dimensionless coordinate along the circumference in the anti-clockwise direction. The furthest right point corresponds to $s=0,1$, and positions $s=0.25$ and 0.75 are located near the posterior median and the umbilicus, respectively. 


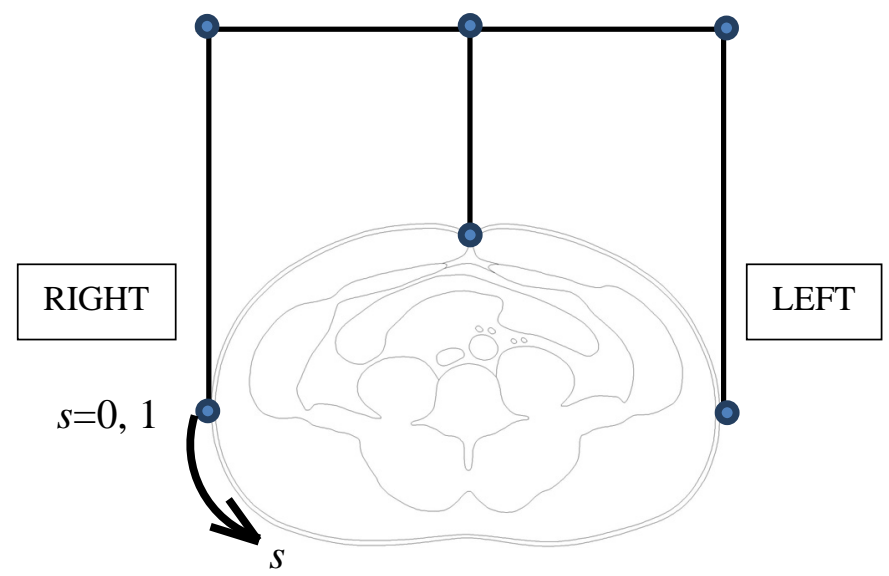

Figure 3: Projection of temperature obtained by thermography onto the circumference of the abdominal cross section. The $s$ is a dimensionless coordinate along the circumference in the anticlockwise direction with the point of origin at the furthest right.

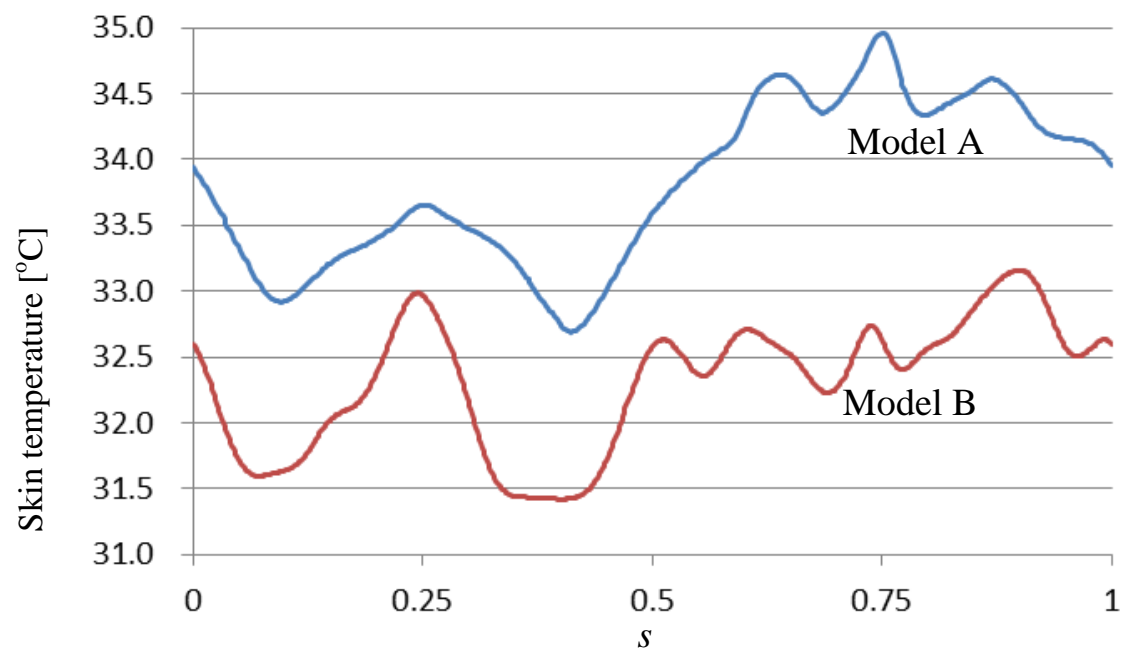

Figure 4: Temperature distributions on the skin surface of models A and B plotted against the dimensionless circumferential length $s$.

It is noteworthy that skin temperature of model B, which has more fat, is lower than A. This is good evidence supporting the discussion in section 2.2. 


\subsection{Approximation to incoming heat $Q_{\text {in }}$}

Incoming heat $Q_{\text {in }}$ consists of (1) heat generation by metabolism, (2) heat transported by blood flow and (3) heat transferred by temperature gradient in the $z$-direction. It is assumed in this study that $Q_{\text {in }}$ is constant within each subdomain. Zero was chosen for $Q_{i n}$ invertebra (subdomain 5) and veins (subdomains 8, 11 and 13).

Reference values for the effects of (1) and (2) were obtained first. Then, the final value for $Q_{\text {in }}$ was calculated by multiplying a factor depending on the model. The factor $f$ was determined so that the total amount of heat released into the air through the skin agreed with the total amount of incoming heat as in the following relation:

$$
\oint_{\Gamma} q_{n} d \Gamma=\int_{S} Q_{i n} d S
$$

where $q_{n}, \Gamma$ and $S$ stand for heat flux normal to the skin surface, the closed curve of the skin surface and the whole area of the abdominal cross section. The lefthand side of eqn (4) can be calculated from the skin heat flux $q_{n}$ which is evaluated from the skin and air temperatures by taking the effects of natural convection and radiation into account.

Physical significance of $f$ is the degree of heat resistance of the abdominal cross section. As discussed in section 2.2, less heat tends to be transferred through a portion of higher heat resistance. The amount of heat released to the air through the abdominal skin surface of model B was smaller than A, although the height and weight shown in Table 1 suggest that more heat is generated in the whole body of B. In other words, a great proportion of heat goes out of the body of $\mathrm{B}$ through portions other than the abdomen. This resulted in a smaller $f$ for $\mathrm{B}$. Thus, the effect of (3) heat transferred by temperature gradient in the $z$-direction can be included in the computational model.

The reference values of (1) and (2) were determined in the following manners.

Gallagher et al. [3] was referred to for benchmark values of heat generated by metabolism per unit time per unit mass. These values are transformed into heat per unit time per volume by multiplying the corresponding densities.

Heat transported by blood flow in capillaries is considered to be proportional to flow rate and temperature gap between blood and the surrounding tissue. Blood flow rate was cited from Raitakari et al. [4] for muscle, Hulten et al. [5] for intestines and Zelis et al. [6] for skin. Blood flow rate determined was approximately $6.5 \mathrm{ml} / \mathrm{m}^{3} / \mathrm{s}$ in intestines while it was $0.5 \mathrm{ml} / \mathrm{m}^{3} / \mathrm{s}$ in muscle and skin. The temperature gap was assumed to be $0.8,0.15$ and $3 \mathrm{~K}$ for muscle, intestines and skin, respectively. The constant of proportionality was determined so that heat brought to the skin region by blood should be approximately 1000 $\mathrm{W} / \mathrm{m}^{3}$ because, otherwise, calculated deep inner temperature of the abdomen would go out of the natural range between 37 and 38 degrees Celsius.

Arteries were treated in a different way because flow rate in arteries is much higher than in capillaries. Heat flux from each artery was calculated with a Nusselt number of 3.66 for laminar flow and a temperature gap of $0.02 \mathrm{~K}$ 
assumed. It should be noted that no dramatic change in the computational result was observed even when different values were chosen for the temperature gap because the arteries occupied a negligibly small part in the whole computational domain.

From the fact that the two volunteers were in the measurement room in which air temperature was kept in a range between 29 and 30 degrees Celsius, metabolism in muscle was considered to be less active than normal [7]. The reference value of $Q_{i n}$ for muscle was, therefore, reduced to the half of that calculated by the approach explained above.

Final values of $Q_{\text {in }}$ after being multiplied by factor $f$ are summarised in Table 2 alongside total heat generated in each domain per unit thickness in the $z$ direction. The heat generated over the whole domain $Q_{a l l}$ is equal to the right hand side of eqn (4).

Table 2: $\quad$ Incoming heat $Q_{\text {in }}\left[\mathrm{W} / \mathrm{m}^{3}\right]$ and the amount of heat generated in each subdomain per unit thickness in the $z$-direction.

\begin{tabular}{|l|c|c|c|c|}
\hline & $Q_{\text {in }}$ & $Q_{\text {in }} \times$ Area & $Q_{\text {in }}$ & $Q_{\text {in }} \times$ Area \\
\hline \hline Factor $f$ & 1.48 & - & 0.80 & - \\
\hline 1. skin & $1780 \mathrm{~W} / \mathrm{m}^{3}$ & $3.1 \mathrm{~W} / \mathrm{m}$ & $962 \mathrm{~W} / \mathrm{m}^{3}$ & $3.1 \mathrm{~W} / \mathrm{m}$ \\
\hline 2, 3. fat & $371 \mathrm{~W} / \mathrm{m}^{3}$ & $2.6 \mathrm{~W} / \mathrm{m}$ & $200 \mathrm{~W} / \mathrm{m}^{3}$ & $8.0 \mathrm{~W} / \mathrm{m}$ \\
\hline 4. muscle & $593 \mathrm{~W} / \mathrm{m}^{3}$ & $9.2 \mathrm{~W} / \mathrm{m}$ & $320 \mathrm{~W} / \mathrm{m}^{3}$ & $7.2 \mathrm{~W} / \mathrm{m}$ \\
\hline 6. intestines & $1666 \mathrm{~W} / \mathrm{m}^{3}$ & $8.7 \mathrm{~W} / \mathrm{m}$ & $900 \mathrm{~W} / \mathrm{m}^{3}$ & $3.8 \mathrm{~W} / \mathrm{m}$ \\
\hline 7. aorta & $7372 \mathrm{~W} / \mathrm{m}^{3}$ & $0.9 \mathrm{~W} / \mathrm{m}$ & $3668 \mathrm{~W} / \mathrm{m}^{3}$ & $0.7 \mathrm{~W} / \mathrm{m}$ \\
\hline 9. fascia & $593 \mathrm{~W} / \mathrm{m}^{3}$ & $0.1 \mathrm{~W} / \mathrm{m}$ & $320 \mathrm{~W} / \mathrm{m}^{3}$ & $0.1 \mathrm{~W} / \mathrm{m}$ \\
\hline 10 SMA & $5348 \mathrm{~W} / \mathrm{m}^{3}$ & $0.1 \mathrm{~W} / \mathrm{m}$ & $2888 \mathrm{~W} / \mathrm{m}^{3}$ & $0.1 \mathrm{~W} / \mathrm{m}$ \\
\hline 12. IMA & $5348 \mathrm{~W} / \mathrm{m}^{3}$ & $0.1 \mathrm{~W} / \mathrm{m}$ & $2888 \mathrm{~W} / \mathrm{m}^{3}$ & $0.1 \mathrm{~W} / \mathrm{m}$ \\
\hline $\begin{array}{l}\text { Total heat } \\
\text { generated } Q_{\text {all }}\end{array}$ & - & $24.8 \mathrm{~W} / \mathrm{m}$ & - & $23.1 \mathrm{~W} / \mathrm{m}$ \\
\hline
\end{tabular}

\subsection{Numerical method}

The boundary element method was applied to the heat conduction equation to obtain numerical solutions. As $\lambda$ and $Q_{i n}$ are constant within each subdomain, the governing equation (3) can be treated as an independent Poisson's equation with a constant term. Conditions of continuity for temperature and heat flux between the neighbouring subdomains were imposed to obtain a solution over the whole computational domain. The number of boundary elements was 2333 in model A and 3007 in model B.

Thermal conductivity of muscle $(\lambda=0.49 \mathrm{~W} / \mathrm{m} / \mathrm{K})$ was cited from Suzuki et al. [8]. A slightly smaller value of $0.001 \mathrm{cal} / \mathrm{cm} / \mathrm{s} / \mathrm{K}(=0.42 \mathrm{~W} / \mathrm{m} / \mathrm{K})$ were used for thermal conductivity of the rest of the other tissues except for fat and vertebra. The subdomain of fat was given a thermal conductivity of $0.21 \mathrm{~W} / \mathrm{m} / \mathrm{K}, 50 \%$ of the conductivity of most other tissues, while $\lambda=0.5 \mathrm{~W} / \mathrm{m} / \mathrm{K}$ was chosen for vertebra. Although a high percentage of calcium containment in vertebra might lead to a higher thermal conductivity, a value comparable to that of muscle was 
adopted because a much higher conductivity than other tissues would give rise to an unusually great concentration of heat in the subdomain of vertebra.

\section{Results and discussion}

Isothermal lines are drawn in Fig.5 from the calculated temperature distributions. Temperature averaged over the subdomain of intestines was 37.7 degrees Celsius in $\mathrm{A}$ and 37.2 degrees Celsius in $\mathrm{B}$, both of which fall into the reasonable range.

It is clearly seen in Fig.5 that a distance between the two neighbouring isothermal lines is shorter in the fat regions than in other portions. This observation agrees with the 1-dimensional theory discussed in section 2.2 that temperature gradient is steeper in a portion of a lower thermal conductivity.

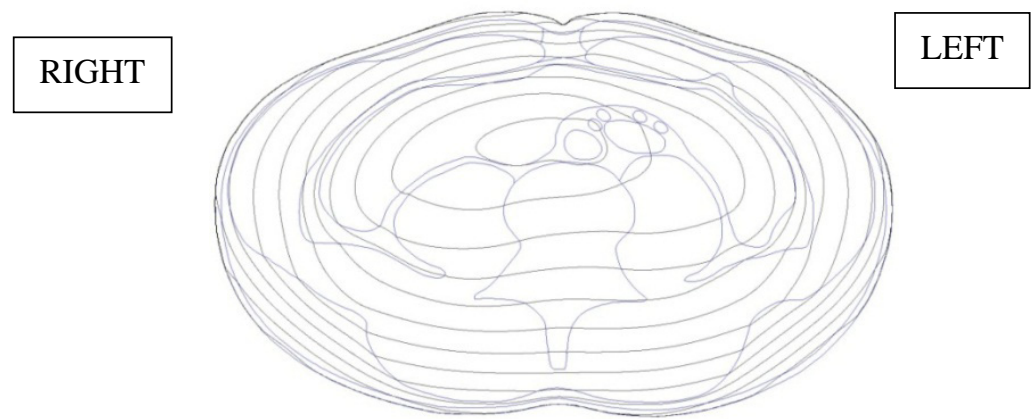

Model A: from 33.0 to 38.5 degrees Celsius

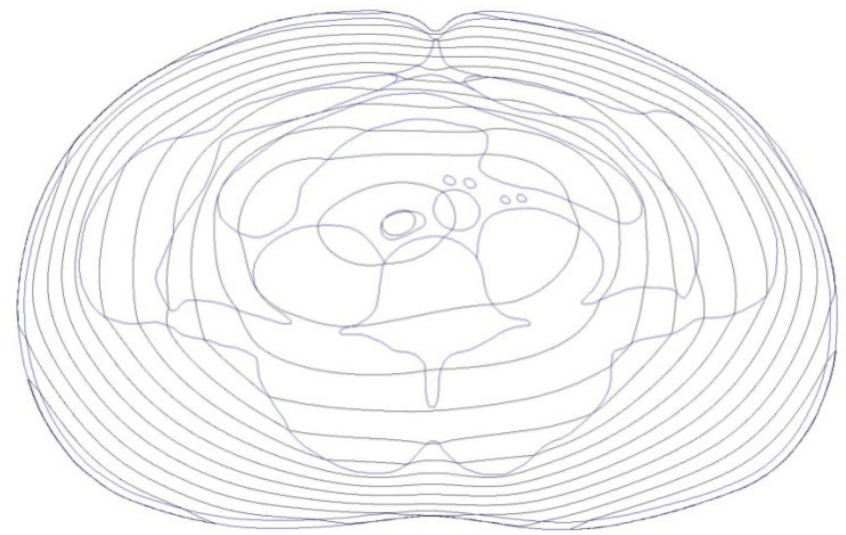

Model B: from 32.0 to 38.5 degrees Celsius

Figure 5: $\quad$ Isothermal lines drawn with an interval of 5 degrees.

Heat flux vector always forms the right angles with isothermal lines. Figure 5, therefore, gives insights into the direction of the heat flow. In this regard, 
curvature of the isothermal lines is instructive. It is obvious from Fig.5 that isothermal lines in the fat regions are characterised by less curvature. This feature is due to the heat resistant effect of the lower thermal conductivity which hinders heat from coming in from other portions.

The heat resistant effect of fat can also be observed in Fig.6 where heat flux vectors are drawn. Heat originating from the intestines goes out of the body through the frontal region of the skin between $\mathrm{P}$ and $\mathrm{Q}(0.44<s<1.09)$ in A. On the other hand, it is noteworthy that heat from the intestines does not reach the posterior half of B. There are two main reasons for this sharp contrast. Firstly, more heat tends to be transferred to a nearer region of the skin. It should be noted that the larger visceral fat area in model B makes the intestines located away from the posterior half. Secondly, visceral fat between the intestines and vertebra interferes with heat transfer in the posterior direction.
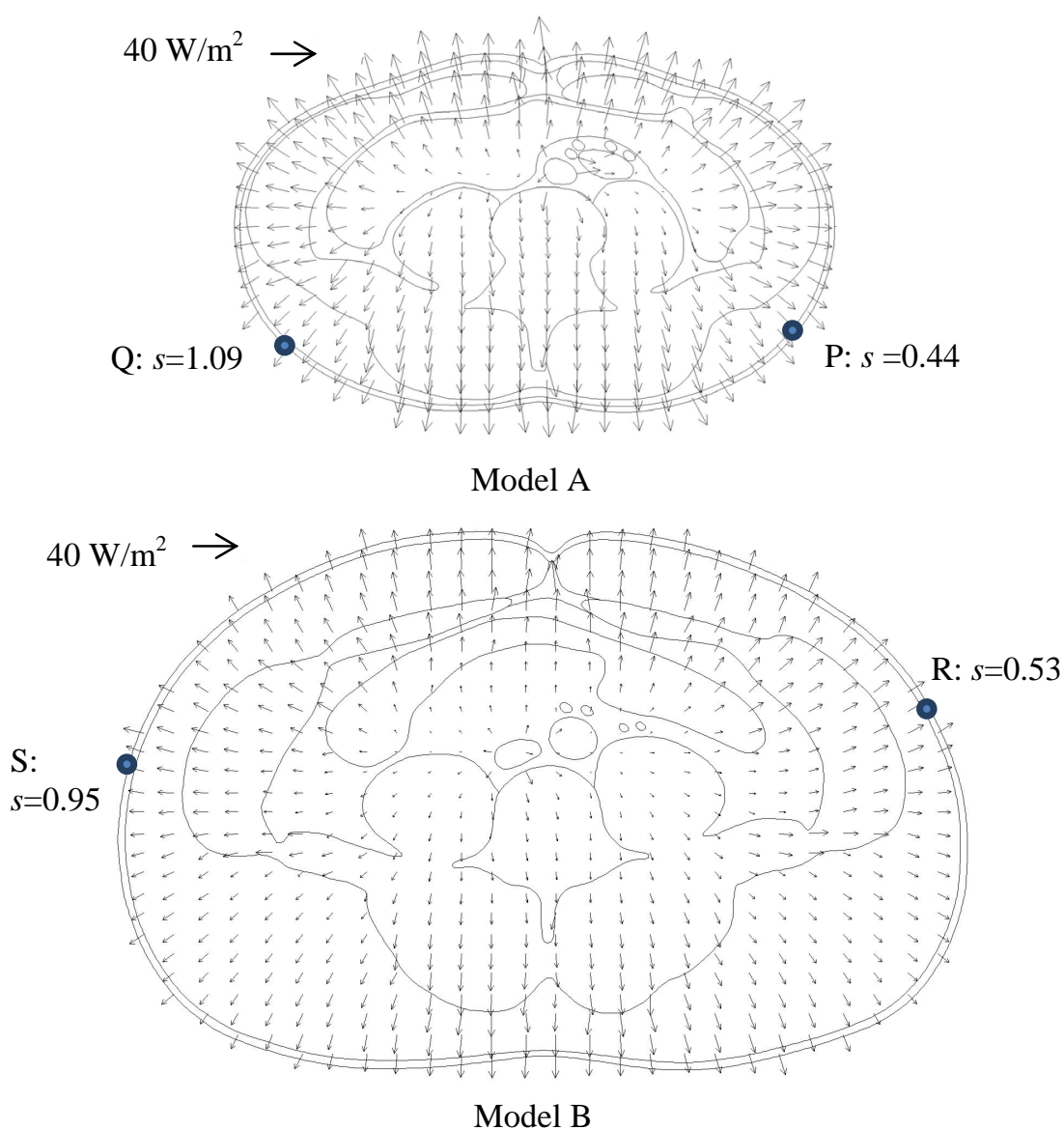

Figure 6: Calculated heat flux vectors. 
It can be concluded from the above discussion that the present computational results strongly suggest a close correlation of the amount of visceral fat with how heat is transferred to the skin and the resultant distribution of skin temperature.

Calculated distributions of heat flux along the skin surface are compared in Fig. 7 with those evaluated by the heat transfer theory in which natural convection and radiation are taken into account. The present heat transfer model can reproduce the overall tendency of the theoretical distributions in both $\mathrm{A}$ and $\mathrm{B}$, although the amount of heat is overestimated in the posterior half $(0<s<0.5)$ and underestimated in the most part of the anterior half $(0.5<\mathrm{s}<1)$. This error can be attributed to too large values of $Q_{\text {in }}$ given to the subdomain of muscle because the muscle makes the greatest contribution to the amount of heat transferred to the posterior half as seen in Fig.6. More accurate calculation can be realised by optimisation of $Q_{\text {in }}$ and $\lambda$. As these two parameters differ from person to person, it seems necessary to collect a great number of samples which can be used as reference data.

\section{Conclusion}

Numerical heat transfer analysis was carried out on two abdominal cross section models with different amounts of fat. The computational results strongly suggested a close correlation of the amount of visceral fat with the state of heat transfer and the resultant distribution of skin temperature. Although more investigation and data accumulation of incoming heat and thermal conductivity are required, there seems to be high feasibility in development of a fat measurement system using temperature distribution on the skin surface.

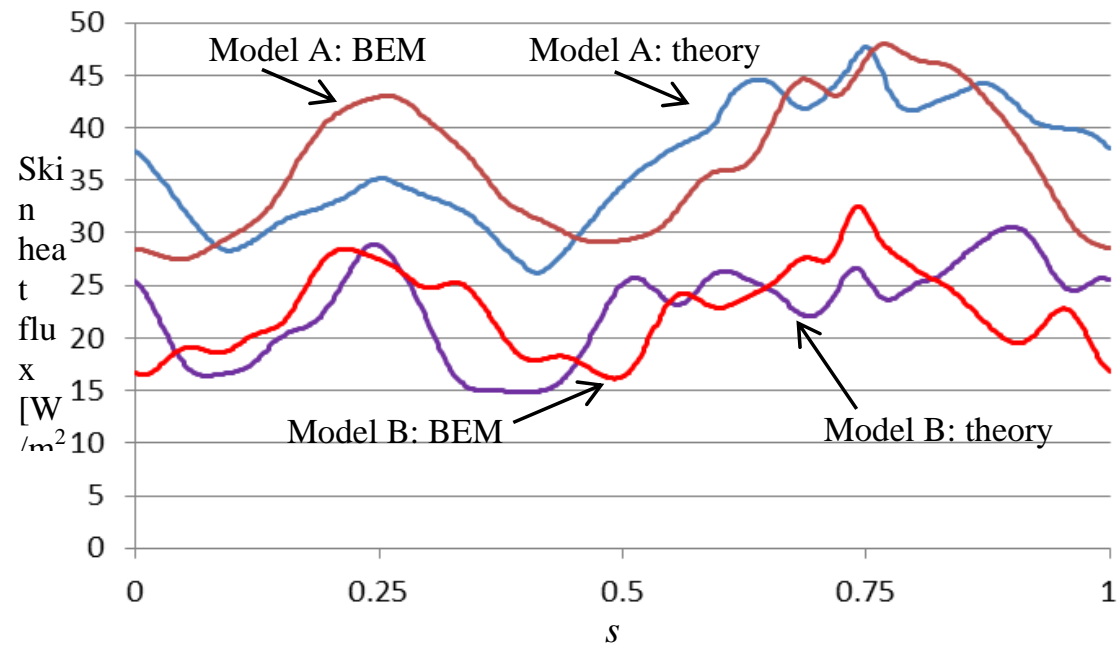

Figure 7: Distributions of skin heat flux along the circumference calculated by BEM and heat transfer theory. 
The authors' final goal is development of a new fat measurement system by putting the present idea into practice. As a future task for realisation of the goal, it is necessary to create an algorithm with which the position and shape of each portion on the abdominal cross section can be calculated from the skin temperature distribution. It is expected that application of the inverse problem theory can enable such calculation.

\section{Acknowledgements}

The authors would like to thank students from Tokyo City University, Mr K Munakata, Mr S Nakajima and $\mathrm{Mr} \mathrm{K}$ Okada, for their cooperation. The remarkable contribution of the two volunteers, who hope to remain anonymous, is also gratefully acknowledged.

\section{References}

[1] Japan Society for the Study of Obesity, Diagnostic Criteria for Obesity(in Japanese), Journal of Japan Society for the Study of Obesity, 17(extra issue), pp. 9-28, 2011.

[2] Ryo, M., Maeda, K., Onda, T., Katashima, M., Okumiya, A., Nishida, M., Yamaguchi, T., Funahashi, T., Matsuzawa, Y., Nakamura, T. and Shimomura, I., A New Simple Method for the Measurement of Visceral Fat Accumulation by Bioelectrical Impedance, Diabetes Care, 28(2), pp. 451453, 2005.

[3] Gallagher, D., Belmonte, D., Deurenberg, P., Wang, Z., Krasnow, N., PiSunyer, F.X. and Heymsfield, S.B., Organ-tissue mass measurement allows modeling of REE and metabolically active tissue mass, American Journal of Physiology, Endocrinology and Metabolism, 275(2), pp. 249-258, 1998.

[4] Raitakari, M. Nuutila, P., Ruotsalainen, U., Teras, M., Eronen, E., Laine, H., Raitakari, O.T., Iida, H., Knuuti, M.J. and Yki-Jarvinen, H., Relationship between limb and muscle blood flow in man, The Journal of Physiology, 496(2), pp. 543-549, 1996.

[5] Hulten, L., Jodal, M., Lindhagen, J. and Lundgren, O., Colonic blood flow in cat and man as analyzed by an inert gas washout technique, Gastroenterology, 70(1), pp. 36-44, 1976.

[6] Zelis, R., Mason D.T. and Braunwald, E., Partition of Blood Flow to the Cutaneous and Muscular Beds of the Forearm at Rest and during Leg Exercise in Normal Subjects and in Patients with Heart Failure, Circulation Research, 24, pp. 799-806, 1969.

[7] Hamaguchi, S., Instrumentation of thermography (in Japanese), The Japanese Journal of Medical Instrumentation, 80(3), pp. 226-233, 2010.

[8] Suzuki, H., Nakayama, M., Otani, T., Kataoka, N. and Tanishita, K., Measurements of Carbon Dioxide Diffusivity and Thermal Conductivity in Muscle Tissue (in Japanese with English abstract), Transactions of the Japan Society of Mechanical Engineers: Series B, 57(535), pp. 1077-1083, 1991. 